\title{
Aufklärungsbögen zu Antidepressiva und Antipsychotika - industrieunabhängig und betroffenenorientiert
}

Peter Lehmann

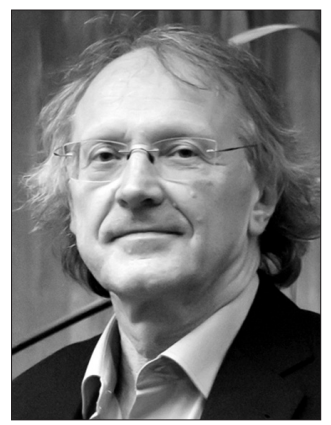

Am 30. November 2018 leiteten PD Dr. med. Anke Brockhaus-Dumke (Chefärztin an der Rheinhessen-Fachklinik Alzey) und Franz-Josef Wagner (Vorsitzender des Netzwerks Selbsthilfe seelische Gesundheit in Rheinland-Pfalz) das Symposium «Wie aufklären über Psychopharmaka?» auf dem Kongress der Deutschen Gesellschaft für Psychiatrie und Psychotherapie, Psychosomatik und Nervenheilkunde e. V. in Berlin. Zu den Teilnehmerinnen und Teilnehmern des Symposiums gehörten Dr. med. Jörg Breitmaier (Chefarzt an der Abteilung für Psychiatrie und Psychotherapie am Krankenhaus Zum Guten Hirten in Ludwigshafen), Dr. med. Sylvia Claus (Chefärztin am Pfalzklinikum vom Pfalzklinikum Klingenmünster) PD Dr. med. Andreas Konrad (Chefarzt an der Allgemeinpsychiatrie und Psychotherapie II der Rhein-Mosel-Fachklinik Andernach) und der Autor dieses Artikels mit seinem Symposiumsbeitrag «Aufklärungsbögen Antidepressiva: Nutzen, Risiken, Absetz- und Entzugsprobleme sowie Alternativen». Wir informierten gemeinsam, wie die beiden Aufklärungsbroschüren des Landesnetzwerks Selbsthilfe seelische Gesundheit Rheinland-Pfalz e. V. (NetzG-RLP) zu Antipsychotika (Neuroleptika) und Antidepressiva zustande gekommen sind, was das Besondere an ihnen ist und wie sie eingesetzt und von Personal und Betroffenen angenommen werden.

\section{Recht auf körperliche Unversehrtheit}

Ziel der Aufklärung ist es, Klinikpatientinnen und -patienten in die Lage zu versetzen, auf Basis industrieunabhängiger Informationen eine eigene Entscheidung zu treffen, ob sie die angebotenen Psychopharmaka einnehmen wollen oder ablehnen. Psychiater und Psychiaterinnen werden unterstützt, über den erhofften Nutzen, die bekannten Risiken und unerwünschten Wirkungen von Psychopharmaka sowie über vorhandene Alternativen aufzuklären. Wenn überhaupt, geschieht die Aufklärung bisher in der Regel unter Zeitdruck und bruchstückhaft oder gar mit falschen Informationen. Aber erst nach einer umfassenden und verständlichen Aufklärung kann man rechtswirksam zustimmen, an- sonsten gilt die Psychopharmaka-Verabreichung als strafbare Körperverletzung.

Bei der Aufklärung geht es nicht etwa um eine gemeinsame Entscheidungsfindung, was oft als Fortschritt gilt. Da das Recht auf körperliche Unversehrtheit, das bei der Verabreichung von Psychopharmaka tangiert ist, ein unteilbares Menschenrecht ist, kann die Entscheidung über ein Ja oder Nein zu Psychopharmaka nicht gemeinsam getroffen, das heisst geteilt werden: Fast wortgleich wie im «Aufklärungsbogen Antipsychotika» steht deshalb auch in der neuen Broschüre:

«Entscheiden Sie sich für die Klinik und die Behandlungsmaßnahme, die Ihren Interessen am ehesten entspricht. Antidepressiva gelten in der Regel als nur eines von mehreren Behandlungsangeboten. Entsprechend der Gesetzeslage und den Behandlungsleitlinien sind es die Patientinnen und die Patienten, die entscheiden, welche Angebote sie annehmen.»

\section{Mehrsprachige Aufklärung}

Beide Broschüren liegen auch in Leichter Sprache vor. Die «Aufklärungsbögen Antipsychotika» gibt es sogar in englischer, französischer, polnischer, spanischer, rumänischer, serbokroatischer, türkischer, russischer und arabischer Übersetzung.

Was die 2018 fertig gewordene Aufklärungsbroschüre zu Antidepressiva betrifft: Diese Substanzen werden bei mittelschweren Depressionen aufgrund von Studienergebnissen nur noch als Alternative zu Psychotherapie und bei schweren Depressionen in Kombination mit Psychotherapie empfohlen. Ihre Wirkung wird nicht mehr mit einem erhöhten Serotonin-Spiegel erklärt; man gesteht ein, dass der Wirkmechanismus unbekannt ist.

Kurz und prägnant sind unerwünschte Wirkungen (sogenannte Nebenwirkungen) aufgelistet: von neurologischen Störungen über Herz-Kreislauf- und Sexualstörungen bis hin zu Aggressivität, erhöhter Suizidalität und plötzlichem Herztod. Weltweit wird erstmals - soweit bekannt - vor dem Risiko körperlicher Abhängigkeit gewarnt; eine Warnung, die inzwischen auch schon einzel- 
ne Pharmafirmen Ärzten und Ärztinnen gegenüber aussprechen.

\section{Unübersehbare Absetz- und Entzugsprobleme}

Man kann es nicht klar genug herausstellen, schliesslich gibt es noch viele Psychiater und Psychiaterinnen der Mainstream-Psychiatrie, die Entzugssymptome in althergebrachter Weise als blosse Anpassungsreaktionen abtun wollen: Mit den «Aufklärungsbögen Antidepressiva» werden Patientinnen und Patienten in psychiatrischen Einrichtungen offen und schon vor Verabreichungsbeginn über das Risiko einer körperlichen Abhängigkeit von Antidepressiva informiert. Das ist ein mutiger Schritt, schliesslich gibt es noch keine Diagnose «Abhängigkeit von Antidepressiva» und diesbezüglich noch keine Finanzierung von Reha-Möglichkeiten. Informiert wird zudem über bestehende Alternativen zu Antidepressiva sowie über - auch kritische - Literatur und weiterführende Internetseiten zu Antidepressiva.
Betroffene, die Antidepressiva absetzen wollen, bekommen Hinweise, welche Absetz- und Entzugssymptome auftreten können und wie man risikovermindernd absetzen kann. Ebenso informiert werden sie über teilweise massive Absetzund Entzugsprobleme sowie Möglichkeiten, dieses Risiko durch vorsichtiges Absetzen zu verringern. Und viele schon jetzt vorhandene Alternativen im Rahmen eines stationären Aufenthalts werden aufgelistet: Psycho-, Wach- und Lichttherapie, therapeutische Gemeinschaft, naturheilkundliche Mittel, Achtsamkeitstraining, kreative Therapien, Sport, Genesungsbegleitung u.v.m. Insbesondere die angebotenen und von Betroffenen geschätzte Sozialberatung zum Beispiel bei Problemen im Bereich Arbeit, Wohnen und Finanzen dürfte einen starken sozial-integrativen Charakter haben.

\section{Win-win-Situation für die Beteiligten}

Mit Ausnahme von Elektroschocks als Alternative zu Antidepressiva, worüber ein unvereinbarer Meinungsunterschied bestand, gelang den Auto-

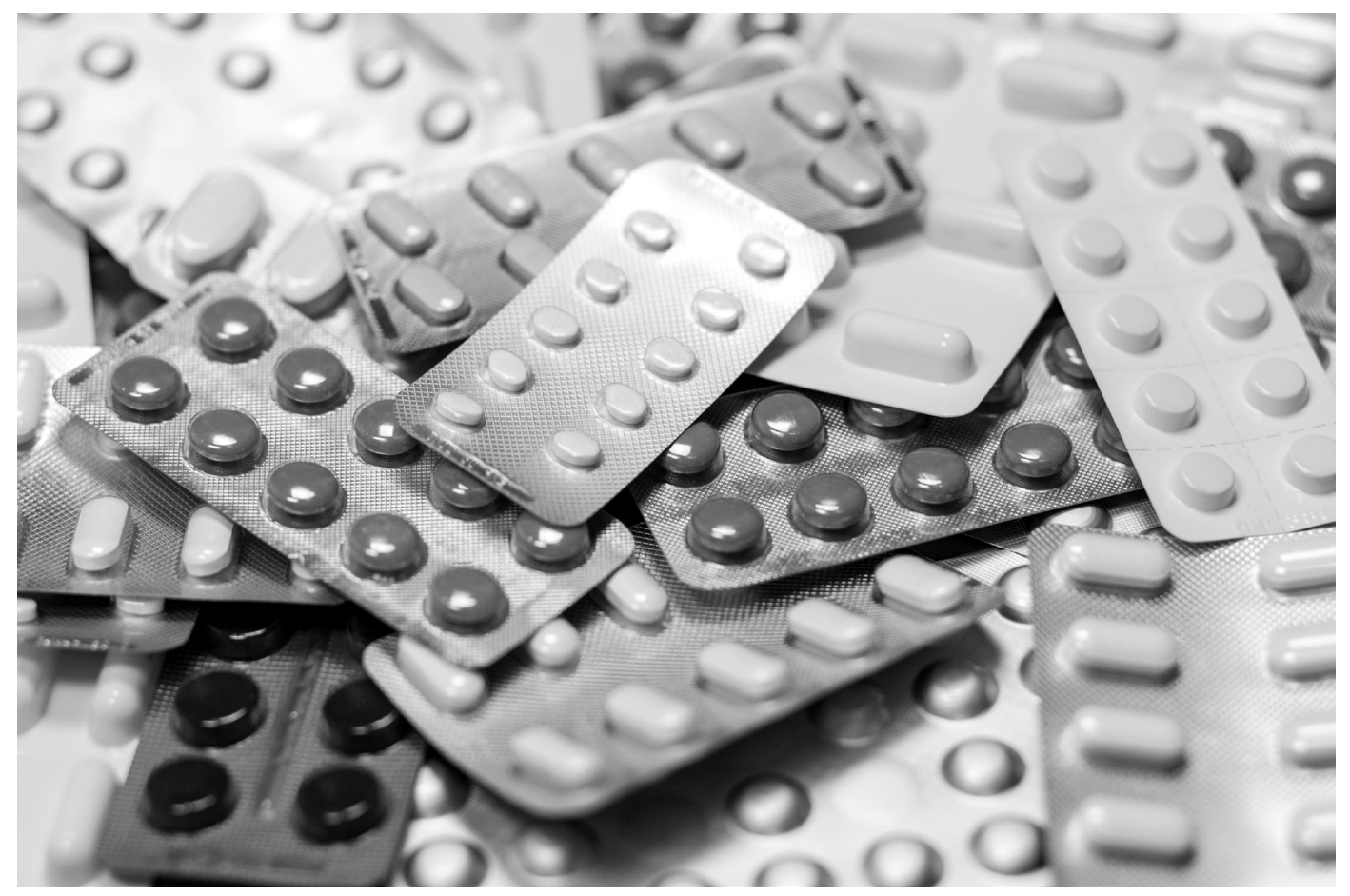


rinnen und Autoren des Aufklärungsbogens nach eigener Einschätzung ein - hoffentlich auch kritischer Betrachtung standhaltendes - überfälliges industrieunabhängiges Dokument. Es unterstützt Anwender und Anwenderinnen von Antidepressiva bei der gesetzlich vorgeschriebenen umfassenden und verständlichen Aufklärung. Und es unterstützt die allfälligen Empfänger und Empfängerinnen in ihrem Recht auf eigene Entscheidung hinsichtlich ihrer körperlichen Unversehrtheit. Somit stellen die Aufklärungsbögen einen wichtigen Schritt in Richtung Gleichheit vor dem Gesetz für Menschen mit psychiatrischen Diagnosen dar. Diese Gleichheit fordert die 2008 in Kraft getretene und in Deutschland Gesetzeskraft ausübende Konvention über die Rechte von Menschen mit Behinderung der Vereinten Nationen.

\section{Nationale und internationale Verbreitung}

Die Verbreitung der Aufklärungsbögen in sozialen Netzwerken und internationalen Mailinglisten erbrachte eine Vielzahl positiver Reaktionen von Psychiatriebetroffenen, Ärzten und Therapeutinnen. Nachfolgend einige ins Deutsche übersetzte Kommentare per E-Mail von engagierten Ärztinnen, Therapeuten und Betroffenen aus aller Welt zu den in diverse Sprachen übersetzten «Aufklärungsbögen Antipsychotika»:

«Vielen Dank für die grossartige Arbeit, die ihr mit der Veröffentlichung dieser Informationsblätter über Neuroleptika geleistet habt» (D. P., USA, 18.12.2018).

«Ich danke für diese Arbeit, die für Menschen mit psychopharmakabedingten schweren körperlichen Leiden so notwendig ist. In meinem Land Argentinien und in der lateinamerikanischen Region werden die verursachten Schäden bestritten. Diese Aufklärungsbögen sind ein wichtiger Beitrag zu den Veränderungen, die wir brauchen» (L. L., Argentinien, 18.12.2019).

«Das sieht sehr praktisch aus. Vielen Dank an Peter und alle Beteiligten» (J. M, England, 18.12.2018).

«Vielen Dank für die bemerkenswerte Informationsbroschüre zu Antipsychotika. Dies ist definitiv ein
Schritt in Richtung vollständiger Offenlegung und informierter Zustimmung» (J. W., Kanada, 19.12.2018).

«Ich stimme Joanna vollkommen zu. Grossartige Leistung und gute Arbeit im Dienste der Seelenheilkunst» (T. I., Schweiz, 3.1.2019).

«Diese Informationen finde ich für litauische Psychiatriebetroffene sehr nützlich, denn solche Informationen gibt es in Litauen nicht. Sie zeigen exemplarisch die Möglichkeiten von Menschen mit psychiatrischen Diagnosen, ihre Situation zu überdenken. Da der Aufklärungsbogen relativ kurz ist, werde ich ihn ins Litauische übersetzen» (L.C., Litauen, 3.1.2019).

«Vielen Dank für all diese wichtige Arbeit. Es muss eine riesige Aufgabe gewesen sein» (K. G., Kanada, 3.1.2019).

«Ich denke, sie sind gut gemacht und dringend nötig» (P.D., USA, 3.1.2019).

«Vielen Dank an Sie und Ihre Kolleginnen und Kollegen für die Bereitstellung wichtiger unabhängiger Informationen über die unerwünschten Wirkungen von Antidepressiva und das zusätzliche Risiko massiver Probleme beim Absetzen dieser Substanzen» (D. E., Kanada, 5.1.2019).

\section{Wo finden Sie die Aufklärungsbögen?}

Alle Aufklärungsbögen sowie Artikel zur gesamten Aktion der Aufklärungsbögen können aus dem Internet unter http://www.peter-lehmann. de/aufklaerung.htm kostenfrei heruntergeladen werden. Bestellen kann man die gedruckten «Aufklärungsbögen Antidepressiva» gegen eine Spende über 3 Euro als Ausgleich für die Versandkosten bei der NetzG-RLP e. V., Franz-Josef Wagner, Gratianstr. 7, D-54294 Trier.

Peter Lehmann, Dipl.-Päd., Dr. phil. h. c. (Aristoteles-Universität Thessaloniki), ist Autor und Verleger in Berlin. Er war bis 2010 langjähriges Vorstandsmitglied des Europäischen Netzwerks von Psychiatriebetroffenen. Mehr unter www.peter-lehmann.de 